\title{
Acetonitrile ingestion: delayed onset of cyanide poisoning due to concurrent ingestion of acetone
}

\author{
M.D. Boggild, R.W. Peck and C.R.V. Tomson \\ Department of Medicine, Leicester Royal Infirmary, Leicester LE2 7LX, UK.
}

\begin{abstract}
Summary: A fatal case of acetonitrile ingestion is reported. The patient presented having apparently taken an overdose but was well until around 24 hours after the supposed ingestion, when cardiovascular collapse and profound metabolic acidosis developed. Later investigation revealed that the patient had taken acetone and acetonitrile. Acetone is known to slow the metabolism of acetonitrile to cyanide, thereby delaying the appearance of toxicity.
\end{abstract}

\section{Introduction}

Acetonitrile (methylcyanide, $\mathrm{CH}_{3} \mathrm{CN}$ ) is widely used as a laboratory solvent, a fumigant, and has also been sold for domestic use. ${ }^{1}$ It is readily absorbed from the lungs and gastrointestinal tract, and is metabolized to cyanide and formaldehyde in the mammalian liver. Despite its known toxicity and wide availability, there are reports of only 2 previous fatalities after ingestion, ${ }^{1,2}$ although several cases of death after accidental inhalation have been reported. ${ }^{3,4}$ Vigorous treatment with the cyanide 'antidotes' sodium thiosulphate, sodium nitrite, hydroxycobalamin and dicobalt edetate has resulted in survival in one case of attempted suicide with acetonitrile. ${ }^{5}$ Acetonitrile itself is non-toxic, and the delayed and gradual appearance of cyanide in the circulation not only allows time for successful treatment but also may obscure the diagnosis. We report a case in which the onset of cyanide poisoning was further delayed by the concurrent ingestion of acetone, which has been shown to inhibit metabolism of acetonitrile.

\section{Case report}

A 22 year old female with a past history of depression and multiple parasuicides was admitted to hospital having been found semiconscious at her place of work. On admission she was conscious

Correspondence: M. Boggild M.B., Ch. B., Leicester General Hospital, Gwendolen Road, Leicester LE5 4PW, UK.

Accepted: 31 July 1989 although drowsy, but refused to give any history; her friends reported that she had probably taken an unknown 'poison' approximately 8 hours before admission. She resisted gastric lavage. Clinical examination was unremarkable apart from a transient sinus tachycardia. Urine drug screen for imipramine, phenothiazines and paracetamol was negative and a serum biochemical screen was normal. She remained drowsy but rousable over the next 18 hours, but then rapidly developed agitation followed by grand mal convulsions and asystolic cardiac arrest. She was resuscitated and transferred to the intensive care unit approximately 26 hours after the supposed ingestion, at which time a bottle marked 'acetone' was found among her belongings.

Immediately after resuscitation she was found to have a profound metabolic acidosis (arterial pH 6.98 , serum bicarbonate $15 \mathrm{mmol} / \mathrm{l}$, chloride $111 \mathrm{mmol} / \mathrm{l}$, sodium $159 \mathrm{mmol} / \mathrm{l}$, potassium $7.0 \mathrm{mmol} / \mathrm{l}$, phosphate $6.28 \mathrm{mmol} / \mathrm{l}$, lactate $36.8 \mathrm{mmol} / \mathrm{l})$. Despite adequate ventilation, treatment with over $450 \mathrm{mmol} / \mathrm{l}$ of sodium bicarbonate, and pressor agents, the patient remained hypotensive (systolic blood pressure $40 \mathrm{mmHg}$ ) and severely acidotic (highest arterial $\mathrm{pH}$ achieved 7.19) and died approximately 30 hours after the supposed ingestion. Later investigation revealed the presence of acetone in trace amounts of blood and in larger quantities in urine, and thin layer chromatography also showed the presence of another, unidentified, volatile solvent. Analysis of the contents of the bottle found with the patient's belongings showed the presence of acetonitrile. Post-mortem examination revealed pulmonary oedema and haemorrhagic gastritis. 


\section{Discussion}

Our purpose in reporting this case is to draw attention to the possibility that acetonitrile ingestion may cause cyanide toxicity, and that this may be delayed considerably due to slow hepatic metabolism. In this case, the patient had also taken acetone, which has been shown in experimental animals to delay the onset of acetonitrile toxicity. ${ }^{6}$ Because of the slow onset of symptoms, cyanide toxicity was not considered at any point in this patient and specific antidotes were not administered. It could be argued that the metabolic acidosis in this patient was due to the effects of cardiac arrest: however, the severity of the acidosis and the lack of response to treatment are clear indicators of a primary metabolic disturbance. The possibility remains that acetone per se may have contributed to the acidosis.

Acetonitrile ingestion should be considered in the differential diagnosis in any patient thought to have taken an 'overdose' who develops metabolic acidosis, even if this occurs over 24 hours after ingestion.

\section{Acknowledgement}

We thank Professor J.D. Swales and Dr M.L. Pepperman for their permission to report this case, the Biochemistry Laboratory of the Leicester Royal Infirmary, and Dr L Brown, Dr C. Kendall, and Mr M. Charman, H.M. Coroner, for their permission to report the forensic and post mortem findings.

\section{References}

1. Caravati, E.M. \& Litovitz, T.L. Pediatric cyanide intoxication and death from an acetonitrile-containing cosmetic. JAMA 1988, 260: 3470-3473.

2. Dequidt, J., Furon, D., Wattel, F. et al. Les intoxications par l'acetonitrile a propos d'un cas mortel. J Eur Toxicol 1974, 7: 91-97.

3. Amdur, M.L. Accidental group exposure to acetonitrile. $J$ Occup Med 1959, 1: 627-633.
4. Willhite, C.C. Inhalation toxicology of acute exposure to aliphatic nitriles. Clin Toxicol 1981, 18: 991-1003.

5. Jaeger, A., Tempe, J.D., Porte, A., Stoeckel, L. \& Mantz, J.M. Acute voluntary intoxication by acetonitrile. Acta Pharmacol Toxicol (Copenh) 1977, 41 (Suppl): 340.

6. Freeman, J.J. \& Hayes, E.P. Acetone potentiation of acute acetonitrile toxicity in rats. $J$ Toxicol Environ Health 1985, 15: 609-621. 